\title{
Differential Evolution Optimization for a Residential Demand Response Application
}

\author{
Ricardo Faia ${ }^{1}$, Fernando Lezama ${ }^{1}$, Pedro Faria ${ }^{1}$, Zita Vale $^{2}$ \\ ${ }^{1}$ GECAD Research Group, Polytechnic of Porto (ISEP/IPP), Porto, Portugal \\ $\{$ rfmfa, flzcl, pnf $\} @$ isep.ipp.pt \\ ${ }^{2}$ Polytechnic of Porto (ISEP/IPP), Porto, Portugal \\ zav@isep.ipp.pt
}

\begin{abstract}
In the smart grid era, when the power system is under stress, demand response (DR) is considered a viable and practical solution for smoothing the demand curve. DR is a procedure that is applied to provide changes in consumers power consumption. These changes can be obtained by optimization techniques producing solutions for the management of power profiles of consumers. In general, optimization techniques can be divided into two groups: the exact methods and the approximate methods. In this paper, an optimization DR problem is formulated and solved using an approximate method based on evolutionary computation. The differential evolution (DE) and one variant called hybrid-adaptive DE (HyDE), as well as the Particle swarm optimization (PSO) algorithms are used and their performance is compared. The results show that $\mathrm{DE}$ algorithms are superior to PSO for this application and their performance is close to that obtained with an exact method.
\end{abstract}

Index Terms-Demand Response, Differential Evolution, Optimization techniques.

\section{INTRODUCTION}

The gap between demand and generation is increasing in the recent decades due the exponential grown in the energy consumption. In the past, power generation obtained using fossil fuels was the option to overcome such situation. However, with the recent trends of the European Commission (EC) for decarbonization of the power system due to climatic concerns, the exploration and implementation of new means for energy generation are increasing. With the massive integration of intermittent generation (new means for energy generation), the power network becomes more exposed to instabilities of generation [1]. The physical equilibrium between load and generation is an issue for power systems operators. The flexibility needed for such equilibrium can be obtained using demand response (DR) control methodologies and smart appliances [2]. DR can be defined as the end users modification of electricity consumption patterns in response to incentives or benefits such as changes in the price of electricity over time.

The application of DR programs to end consumers has been done over time through an aggregator. In this scenario, the aggregator works as a service provider to whom the DR

The present work was done and funded in the scope of the following projects: SPEAR Project, CEECIND/02887/2017, UID/EEA/00760/2019 funded by FEDER Funds through COMPETE program and by National Funds through FCT and PhD grant SFRH/BD/133086/2017. services must be paid. A thermostatically controlled loads in aggregation mode is presented in reference [3] for doing DR. The applications without contracts with services provider (standalone application) is also possible [4], [5]. In [6], the standalone application of DR in a residential house (i.e., an independent user with specialized equipment to perform DR) is considered. The optimization is performed for a 24-hour horizon with 15-minute periods. The solution presented optimizes the loads that must perform DR (load cut only) and indicates the amount of charge and discharge that the batteries must carry out. Thus, it is expected that the user will minimize the costs of operation and can sell electricity to the network.

Considering the reasons that the authors of the publication [6] described to justify the use of metaheuristics in this type of applications, this paper propose the use of differential evolution (DE) for DR optimization considering a standalone application. $\mathrm{DE}$ is arguably one of the most powerful evolutionary algorithms in current use [7]. The performance of DE is compared with another well-known metaheuristic, the particle swarm optimization (PSO). Results show that DE outperforms PSO in the presented problem and achieves near-optimal solutions close to a deterministic solution obtained through the use of CPLEX. Thus, it is demonstrated that the use of metaheuristics is feasible in this type of problems and can contribute to a better economy of the electricity end-user.

This paper is divided into five section. The introduction is presented in this section, Section II present the problem with the mathematical formulation and the solution encoding for metaheuristic implementation. Section III presents the DE algorithm, and also a new variant called hybrid-adaptive DE (HyDE). Section V present the case study and numerical results, a conclusion of the work is presented in last section.

\section{PROBEM FORMULATION}

This section is divided into two parts. Initially, the mathematical formulation is presented with all variables as equation that are applied in the optimization method. The encoding of individuals (i.e., the representation of solutions in the form of vectors) is presented in the second part. 


\section{A. Mathematical formulation}

The presented optimization model is considered a mixedinteger linear problem (MILP). Equation (1) represents the objective function:

\section{Minimize $f=$ Energy Bill $+D R$ Curtailment}

Equation (1) include the sum of two equations: the energy bill presented in equation (2) and the DR curtailment presented in equation (3). Equation (2) shows the buying and selling energy costs, and equation (3) represents the cost weighting combined with $\mathrm{kWh}$ curtailment.

In energy bill equation the variable $P_{t}^{\text {grid }}$ signifies the energy flow between grid and household, $I_{t}^{\text {grid in }}$ is a variable that indicates power flow into the grid and control the energy buy $\left(I_{t}^{\text {grid in }}=1\right)$ and energy sell $\left(I_{t}^{\text {gridin }}=0\right), C_{t}^{\text {grid in }}$ represents the cost of buying electricity and $C_{t}^{\text {grid out }}$ represents the cost of selling electricity. The energy bill in equation (2) considers the costs of buying electricity $\left(I_{t}^{\text {grid in }} \times P_{t}^{\text {grid }}\right) \times$ $C_{t}^{\text {grid in }}$ and the revenues of selling electricity $\left(\left(1-I_{t}^{\text {grid in }}\right) \times\right.$ $\left.P_{t}^{\text {grid }}\right) \times C_{t}^{\text {grid out }}$. In each period $(t)$ the user can make a single operation (buy or sell).

$$
\begin{aligned}
& \text { Energy Bill }=\sum_{t=1}^{T}\left[\left(\left(I_{t}^{\text {grid in }} \times P_{t}^{\text {grid }}\right) \times C_{t}^{\text {grid in }}\right.\right. \\
& -\left(\left(1-I_{t}^{\text {grid in }}\right) \times P_{t}^{\text {grid }}\right) \\
& \left.\left.\times C_{t}^{\text {grid out }}\right) \times \frac{1}{\Delta t}\right]+D C P \\
& I_{t}^{\text {grid in }}= \begin{cases}1, & \text { if } P_{t}^{\text {grid }}>0 \\
0, & \text { otherwise }\end{cases} \\
& \in\{1, \ldots, T\}
\end{aligned}
$$

Also in equation (2) the term $\left(\left(1-I_{t}^{\text {grid in }}\right) \times P_{t}^{\text {grid }}\right)$ represents the power sent to the network. The term $\Delta t$ is applied for to adapt the consumption to the tariff price because typically the tariff is presented in EUR/kWh and the optimization can be arranged at different time intervals (e.g. 15 minutes). $D C P$ represent the daily contracted power cost. This variable $P_{t}^{\text {grid }}$ if in optimization take positive values means that there is electricity consumption from the network. However, if takes negative values means that there is a sale of electricity to the network. Equation (3) presents the DR curtailment.

$$
\text { DR Curtailment }=\sum_{t=1}^{T}\left(\sum_{l=1}^{L} P_{l, t}^{\text {cut }} \times X_{l, t}^{\text {cut }} \times W_{l, t}^{\text {cut }}\right)
$$

In equation (3) is applied the load cut cost with the use of weights, that, in fact, has no cost for the user. The variable $P_{l, t}^{\text {cut }}$ is the of load $(l)$ cut energy in period $(t)$, the $X_{l, t}^{\text {cut }}$ signifies the decision binary variable to active the cut of load $(l)$ in period $(t)$ and $W_{l, t}^{\text {cut }}$ signifies the load $(l)$ cut weight in period $(t)$. The component $\left(P_{l, t}^{c u t} \times X_{l, t}^{c u t} \times W_{l, t}^{c u t}\right)$ indicates the user interest to make cut in load $(l)$ in period $(t)$.
Equation (4) represents the balance between load and generation, $P_{b, t}^{b a t}$ represents the energy charged or discharged by baterry $(b)$ in period $(t)$. If the value of $P_{b, t}^{b a t}$ is less than 0 means the battery is discharging, otherwise, if the value of $P_{b, t}^{b a t}$ is greater than 0 means battery is charging. The variable $P_{p, t}^{P V}$ represents the photovoltaic production of unit $p$ at period $t$, and $P_{t}^{\text {load }}$ corresponds to the value of load at period $t$.

$$
\begin{aligned}
P_{t}^{\text {grid }}=P_{t}^{\text {load }}+ & \sum_{b=1}^{B} P_{b, t}^{\text {bat }}-\sum_{l=1}^{L} P_{l, t}^{\text {cut }} \times X_{l, t}^{\text {cut }} \\
& -\sum_{p=1}^{P} P_{p, t}^{P V}, \quad \forall t \in\{1, \ldots, T\}
\end{aligned}
$$

The balance of battery systems is presented in equation (5).

$$
\begin{aligned}
E_{b, t}^{s t o r}=E_{b, t-1}^{s t o r}+ & P_{b, t}^{\text {bat }} \times \frac{1}{\Delta t}, \\
\forall t & \in\{2, \ldots T\}, \forall b \in\{1, \ldots, B\}
\end{aligned}
$$

Variable $E_{b, t}^{s t o r}$ represents the state of the battery $b$ in period $t$, in other words, represent the amount of energy it has available. The current battery state in equation (5) is achieved by adding the anterior state $E_{b, t-1}^{s t o r}$ to the $P_{b, t}^{b a t}$ variable. The power term $P_{b, t}^{b a t}$ in equation (5) is multiplied by $\frac{1}{\Delta t}$ to convert power into energy units. The system is governed by the following constraints (equation (6) to (10)).

$$
\begin{aligned}
& -P_{t}^{\text {grid min }} \leq P_{t}^{\text {grid }} \leq P_{t}^{\text {grid max }} \quad \forall t \\
& \in\{1, \ldots, T\} \\
& P_{l, t}^{\text {cut }}=P_{l, t}^{\text {cutmax }} \quad \forall l \in\{1, \ldots, L\}, \forall t \in\{1, \ldots, T\} \\
& 0 \leq E_{b, t}^{\text {stor }} \leq E_{b, t}^{\text {stor max }} \quad \forall b \in\{1, \ldots, B\}, \forall t \\
& \in\{1, \ldots, T\} \\
& -P_{b, t}^{d c h \max } \leq P_{b, t}^{b a t} \leq P_{b, t}^{c h \max } \quad \forall b \\
& \in\{1, \ldots, B\}, \forall t \in\{1, \ldots, T\} \\
& X_{l, t}^{c u t}=\left\{\begin{array}{l}
1 \\
0
\end{array} \quad \forall l \in\{1, \ldots, L\}, \forall t \in\{1, \ldots, T\}\right.
\end{aligned}
$$

In equation (6) the variable $P_{t}^{\text {grid min }}$ and $P_{t}^{\text {grid max }}$ represents the limit values for variable $P_{t}^{\text {grid }}$. Equation (7) identify that the $P_{l, t}^{c u t}$ can only take the maximum value $P_{l, t}^{\text {cut max }}$. The $P_{b, t}^{b a t}$ variables can take a value between $-P_{b, t}^{d c h \text { max }}$ and $P_{b, t}^{c h \text { max }}$, if the value of $P_{b, t}^{\text {bat }}$ is less than zero represents a discharge and if greater than zero represents a charge. The variable $X_{l, t}^{\text {cut }}$ is a binary variable and represents a decision variable. When $X_{l, t}^{\text {cut }}$ is equal to 1 the cut of load $(l)$ at period $(t)$ is active.

\section{B. Encoding}

The encoded vector presented in equation (11) is used for resolving the problem of section 2 .

$$
\vec{x}_{i}^{j}=\left[\left\{P_{1,1}^{b a t}, \ldots, P_{B, T}^{b a t}\right\},\left\{X_{1,1}^{c u t}, \ldots, X_{L, T}^{c u t}\right\}\right]
$$

where, $\left\{P_{1,1}^{b a t}, \ldots, P_{B, T}^{b a t}\right\}$ is a continuous variables set that correspond to the electricity quantity of discharge or charge in 
battery $(b)$ at period $(t),\left\{X_{1,1}^{c u t}, \ldots, X_{L, T}^{c u t}\right\}$ are binary variables to enable the possibility of performing cut action in load $(l)$ at period $(t)$. Therefore, a particle/individual $\vec{x}$ has a dimension $n=B \times T+L \times T$. This encoding permits obtain the objective value in equation (1).

The implementation of evolutionary algorithms starts by defining the search space limits setting the lower and upper bounds of each variable. In equation (12), $x l b^{j}$ represent the lower limits for the $j$ th solution, and $x u b^{j}$ in equation (13) represent the upper limit for the $j$ th solution (a solution is called individual in DE and particle in PSO).

$$
\begin{aligned}
& x l b^{j} \\
& =\left[\left\{-P_{1,1}^{d c h \max }, \ldots,-P_{B, T}^{d c h} \max \right\},\left\{X_{1,1}^{\text {cut min }}, \ldots, X_{L, T}^{\text {cut min }}\right\}\right] \\
& x u b^{j}=\left[\left\{P_{1,1}^{c h \max }, \ldots, P_{B, T}^{c h} \max \right\},\left\{X_{1,1}^{\text {cut } \max }, \ldots, X_{L, T}^{\text {cut } \min }\right\}\right] \\
& \vec{x}_{1}^{j}=\operatorname{rand}\left[x l b^{j}, x u b^{j}\right]
\end{aligned}
$$

Equation (14) presents the process of initialization where the initial solutions are created. In this process, a random method within permitted limits is performed. $\operatorname{rand}\left[x l b^{j}, x u b^{j}\right]$ is a random number within the lower $x l b^{j}$ and the upper $x u b^{j}$ bounds of $j$ particle for $n$ variables.

\section{DIFFERENCIAL EVOLUTION AND HYDE}

In this section, we briefly introduce the evolutionary algorithms used, differential evolution and Hybrid adaptive DE, to solve the optimization problem of this paper.

\section{A. Differential evolution}

In evolutionary algorithms literature differential evolution (DE) is one of the most efficient and utilized [7], [8]. It can be applied to maximize or minimize any given multi-dimensional function $f\left(x_{1}, x_{2}, \ldots, x_{D}\right)$, where $D$ is the dimension of the problem (i.e., number of variables to optimize). The standard DE algorithm uses a population (Pop) of individuals representing solutions to a given problem $\vec{x}_{j, i, G}=$ $\left[\vec{x}_{1, i, G}, \vec{x}_{2, i, G}, \ldots, \vec{x}_{D, i, G}\right]$, where $G$ is the generation number, and $i=[1,2, \ldots, N P]$ is the number of individuals in the population. At each iteration $G$, all individuals $\vec{x}_{i, G} \in$ Pop are evaluated in a fitness function. The individual being measured is named the goal vector $\left(\vec{x}_{i, G}\right)$. For each target vector $\vec{x}_{i, G}$, a mutant individual $\vec{m}_{i, G}$ is generated using the mutation operator:

$$
\vec{m}_{i, G}=\vec{x}_{r 1, G}+F\left(\vec{x}_{r 2, G}-\vec{x}_{r 3, G}\right)
$$

where $\vec{x}_{r 1, G}, \vec{x}_{r 2, G}, \vec{x}_{r 3, G} \in$ Pop are three random individuals mutually different and also different from the current target vector $\vec{x}_{i, G}$, and the $F$ parameter is known as the mutation constant $(F \in[0,1])$, which controls the mutation strength.

After creating the mutant vector, the recombination operator is used to generate the trial individual as follows:

$$
\begin{aligned}
& \vec{t}_{i, G} \\
& =\left\{\begin{array}{cc}
\vec{m}_{j, i, G} & \text { if }\left(\operatorname{rand}_{i, j}[0,1]<\operatorname{Cr} \vee(j=R n d)\right. \\
x_{j, i, G} & \text { otherwise }
\end{array}\right.
\end{aligned}
$$

Particularly, for each component $j$, where $\mathrm{j}=[1,2, \ldots, D]$, the $j$ th element of $\vec{m}_{j, i, G}$ is chosen with probability $\mathrm{Cr}$, otherwise is chosen from $x_{j, i, G}$. Additionally, a random integer value Rnd with the bonds $[1, \mathrm{D}]$ is utilized to ensure that at least one component is taken from $\vec{m}_{j, i, G} . C r$ is known as the recombination constant $(\mathrm{Cr} \in[0,1])$ and is used to increase the diversity in the mutation process.

One step of paramount importance after creating $\vec{t}_{i, G}$ is the variables boundary constraints verification to avoid creating infeasible solutions. If some elements of the test vector violate the limits, they must be repaired with a random number within the allowable limit or with any other technique such as backbounce or boundary reinitialization [7].

The final step in the iterative process of DE is the selection procedure. In the simple $\mathrm{DE}$, this is reached by a naive elitist rule making the comparison of the fitness between the trial vector $\vec{t}_{i, G}$ and the target vector $\vec{x}_{i, G}$ :

$$
\operatorname{Pop}_{i, G+1}=\left\{\begin{array}{cc}
\vec{t}_{i, G} & \text { if } f\left(\vec{t}_{i, G}\right) \leq f\left(\vec{x}_{i, G}\right) \\
x_{j, i, G} & \text { otherwise }
\end{array}\right.
$$

The $P o p_{i, G+1}$ in the equation (7) represent the population of the next generation, that changes by rejecting or accepting new individuals, and $f($.$) is the fitness function used to measure the$ performance of an individual (i.e., Eq. (1)).

This is the most basic DE mutation strategy also known as $\mathrm{DE} / \mathrm{rand} / 1$. The process is repeated for a fixed number of generations (GEN) until a satisfactory solution is obtained or a computational condition is reached [9]. Throughout the execution process, the user defines $F, C r$, and $N P$. These parameters are maintained fixed throughout the execution of the algorithm. Several studies have shown the impact that the selection of such parameters have in the quality of the solutions [10] and [11]. This is solved in some cases by performing a preliminary phase of tuning to determine the best set of parameters for each problem (see for instance [12], in which a parameter tuning has been done to solve this problem using PSO). To avoid this parameter tuning, adaptive parameter control mechanisms has been proposed to determine such set of parameters without involving previous knowledge of the problems to be solved. In the next section, we briefly introduce a parameter self-adaptive version of DE proposed in [13].

\section{B. Hybrid Adaptive Differential Evolution $(H y D E)$}

HyDE is a new self-adaptive version of DE proposed in [13]. HyDE strategy is known as "DE/target-toperturbed_best/1" and combines the well-known DE/target-tobest/1 strategy with a perturbation of the best individual (inspired by the evolutionary PSO [14]), and the self-adaptive mechanism of $\mathrm{jDE}$ [15]. The main operator of HyDE is defined as follows:

$$
\begin{aligned}
\vec{m}_{i, G}=\vec{x}_{i, G}+F_{i}^{1}(\varepsilon & \left.\cdot \vec{x}_{\text {best }}-\vec{x}_{i, G}\right) \\
& +F_{i}^{2}\left(\vec{x}_{r 1, G}-\vec{x}_{r 2, G}\right)
\end{aligned}
$$

where $F_{i}^{1}$ and $F_{i}^{2}$, are scale factors in the range $[0,1]$ independent for each individual $i$, and updated each iteration following the same rule of jDE algorithm (see Sect. III.B of [13]). The HyDE algorithm applies a perturbation method of the $\vec{x}_{\text {best }}$ :

$$
\varepsilon=N\left(F_{i}^{3}, 1\right)
$$


where the $\vec{x}_{\text {best }}$ in the population is multiplied with a random number obtained from a normal distribution with mean $F_{i}^{3}$, which is also an adaptive parameter updated utilized the equal rule of jDE. Such perturbation is inspired in EPSO [14] and has proven to improve the convergence capabilities of the algorithms when solving optimization problems in the energy domain. HyDE also update $\mathrm{Cr}$ parameter using the same rule of jDE algorithm.

\section{NUMERICAL RESULTS AND DISCUSSION}

This section is divided into two subsections. First the case study is present and second are present the numerical results obtained with the application the mathematical formulation to the case study presented.

\section{A. Case study}

A Portuguese consumer is considered for case study, he has a time of use tariff with three different periods in a day. In the period of peak $(10.30 \mathrm{~h}-13 \mathrm{~h}$ and $19.30 \mathrm{~h}-21 \mathrm{~h})$ the price for electricity buy is $0.2738 \mathrm{EUR} / \mathrm{kW}$, in intermediate period $(08 \mathrm{~h}$ $-10.30 \mathrm{~h}, 13 \mathrm{~h}-19.30 \mathrm{~h}$ and $21 \mathrm{~h}-22 \mathrm{~h}$ ) the price is 0.1572 EUR/kW and in a off-peak $(22 \mathrm{~h}-02 \mathrm{~h}$ and $02 \mathrm{~h}-08 \mathrm{~h})$ period the price is $0.1038 \mathrm{EUR} / \mathrm{kW}$ [16]. The user has the possibility of sell electricity to the grid and the price is $0.1659 \mathrm{EUR} / \mathrm{kW}$, the day fees of contract is $0.5258 \mathrm{EUR} / \mathrm{day}$. The variable $W_{l, t}^{\text {cut }}$ can take the values of 0 in peak periods, 0.2 in intermediate periods and 0.4 in off peak periods.

The maximum power injected to the grid is $-5.1 \mathrm{~kW}$, the maximum power required to the grid is $1000 \mathrm{~kW}$, the maximum power accumulated to the battery is $12 \mathrm{~kW}$ the maximum energy of battery discharge is $-6 / 4 \mathrm{kWh}$, the maximum energy of battery charge is $6 / 4 \mathrm{kWh}$. The total of periods is 93 , have 3 loads that the control is possible, the total of batteries is 1 and the PV units is 2 , the parameter $\Delta t$ is 4 .

\section{B. Numerical results}

We have implemented the basic DE, the adaptive HyDE, and the PSO algorithms, and compared their performance solving the case study introduced in Sect. V.A. The parameters used for each algorithm are provided in Tables I and II. For DE, we have used the recommended parameters in the literature [7]. HyDE is a self-adaptive version, but we have initialized the parameters values with the same recommended values of DE.

TABLE I. DE AND HYDE PARAMETERS USED.

\begin{tabular}{|l|c|c|}
\hline \multicolumn{1}{|c|}{ Parameters $^{\mathrm{a}}$} & Symbol & Value \\
\hline Population size & NP & 500 \\
\hline Number of iterations & Gen & 500 \\
\hline Mutation constant & $F$ & 0.5 \\
\hline Recombination constant & $\mathrm{Cr}$ & 0.9 \\
\hline Number of evaluations & - & 250000 \\
\hline Number of trials & - & 30 \\
\hline
\end{tabular}

a. HyDE adapts the value of $F$ and $C r$ over iterations, but these initial values have been used

For PSO, we have used the set of parameters reported in [6], since those values were obtained after a tuning phase for this specific problem. Concerning the length of the population (or swarm in the PSO), we performed two different simulations, one with a population of 500 individuals (since this was the value with the best performance in [6]) and a second test reducing the population to 10 individuals. The reported values are the average of 30 trials. The tests were performed utilizing MATLAB 2014b 64 bits in a computer with Intel Xeon(R) E52620v2@2.1 GHz processor with $16 \mathrm{~GB}$ of RAM running windows 10.

TABLE II. PSO PARAMETERS USED.

\begin{tabular}{|l|c|c|}
\hline \multicolumn{1}{|c|}{ Parameters } & Symbol & Value \\
\hline Swarm size (Population) & $j^{\text {max }}$ & 500 \\
\hline Number of iterations & $i^{\text {max }}$ & 500 \\
\hline Inertia weight & $w^{\text {min }}-w^{\text {max }}$ & $0.4-0.9$ \\
\hline Cognitive weight & $c_{1}^{\text {min }}-c_{1}^{\text {max }}$ & $0.5-1.5$ \\
\hline Global weight & $c_{2}^{\min }-c_{2}^{\text {max }}$ & $0.5-1.5$ \\
\hline Number of evaluations & - & 250000 \\
\hline Number of trials & - & 30 \\
\hline
\end{tabular}

The controllable and non-controllable loads represents the load consumption, the discharge and charge represents the battery behavior and the load consumption plus battery charges represent the final load, all of these variables are presented in Fig. 1.

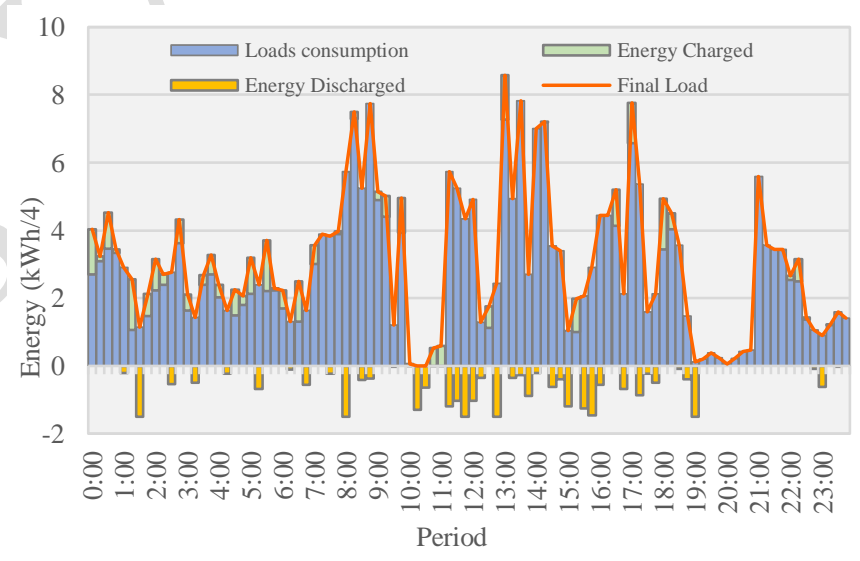

Fig. 1 - Schedule of final load, load consumptions and battery behavior

Fig. 1 demonstrates that when the generation exceeds the consumption requirements, in this scenario, the energy surplus will be used to sell in grid or charge the battery. As follow, the user escapes buying energy from the grid to meet consumption needs and to charge the battery. The battery discharge actions are mostly making between 11.00 and 21.00 hours that represent to a peak and intermediate period.

Table III shows the average fitness and standard deviation obtained with the tested methods with a population of 500 individuals/particles. It can be seen that PSO performs slightly worse than the two variants of DE. Also, it is interesting to see that DE obtains the best average performance (even better than the adaptive HyDE), which indicates that the selected parameters (corresponding to the recommended values in the literature) are in fact a good choice for this particular problem. 
TABLE III. RESULTS USING 500 INDIVIDUALS AND 500 ITERATIONS

\begin{tabular}{|c|c|c|c|c|c|c|}
\hline & & & & Best solution found & & \\
\hline Method & Average fitness & Std & Minimum Cost (EUR) & Energy bill (EUR) & DR revenues (EUR) & Average time (seconds) \\
\hline PSO [6] & 3.5131 & 0.1452 & 3.2909 & 5.9793 & 2.6884 & 153.3833 \\
\hline DE & 3.2658 & 0.0053 & 3.2556 & 6.3105 & 3.0550 & 141.4633 \\
\hline HyDE & 3.3061 & 0.0107 & 3.2764 & 5.8502 & 2.5738 & 139.6367 \\
\hline CPLEX & - & - & 3.1874 & 6.9380 & 3.7505 & - \\
\hline
\end{tabular}

TABLE IV. RESULTS USING 10 INDIVIDUALS AND 500 ITERATIONS

\begin{tabular}{|c|c|c|c|c|c|c|}
\hline & & & & Best solution found & & \\
\hline Method & Average fitness & Std & Minimum Cost (EUR) & Energy bill (EUR) & DR revenues (EUR) & Average time (seconds) \\
\hline PSO [ref] & $\begin{array}{r}4.7128 \\
\end{array}$ & 0.5256 & 3.9695 & 6.1149 & 2.1454 & 5.4053 \\
\hline DE & 3.8218 & 0.2486 & 3.4207 & 5.9209 & 2.5002 & 2.9210 \\
\hline HyDE & 3.6184 & 0.1783 & 3.3593 & 5.8337 & 2.4744 & 3.0267 \\
\hline MILP & - & - & 3.1874 & 6.9380 & 3.7505 & - \\
\hline
\end{tabular}

In Table IV, we have reduced the size of the population to only 10 individuals/particles to see the impact of this reduction in the quality of the solutions. As can be seen, the performance of all algorithms deteriorates with this modification, but the optimization becomes faster, requiring also some seconds to obtain a near-optimal solution. This is important when largescale instances of the problem need to be solved. Again, PSO got the worst performance, while this time HyDE provided the best average fitness from the tested algorithms.

We have also analyzed the convergence characteristics of the algorithms. To that end, Fig. 2 shows the average convergence, with the minimum and maximum bounds of the 30 trials.

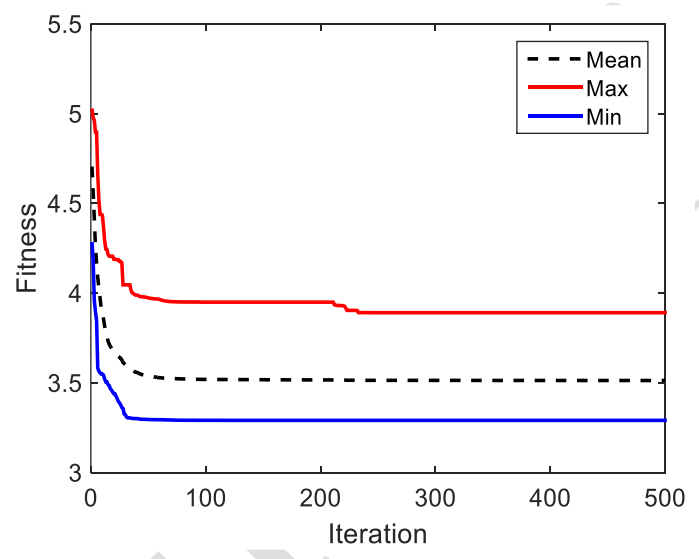

a)

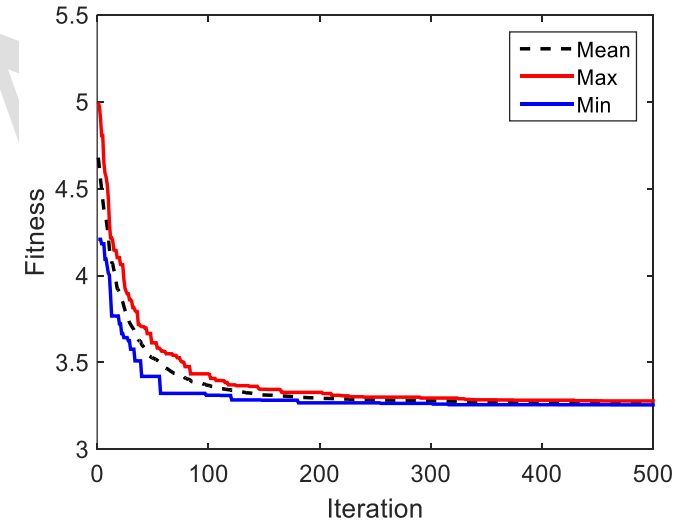

b)

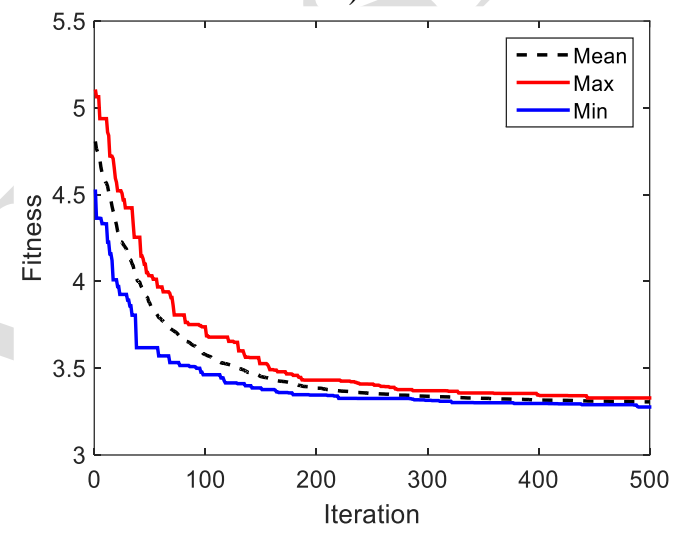

c)

Fig. 2 - Convergence behivor of the tested algorithms with a population of 500 individuals. a) PSO. b) DE. C) HyDE.

The PSO algorithms has a less robust behavior, obtaining a higher standard deviation, as can be seen in Fig. 2. On the other hand, DE and HyDE showed a very similar convergence behavior despite HyDE adapts the parameters in the long of the iterations. This indicates that the selection of recommended parameters for the standard DE is actually a good choice for this specific problem.

The convergence characteristics has been also analyzed for the case of fewer individuals (i.e., 10 individuals/particles) in Fig. 3. In this case, all the algorithms presented less robustness over the 30 performed trials, by showing a marker gap between the average and min/max values. PSO again had the worst performance of the three tested algorithms. Also, it can be seen that while DE shows a behavior of stagnation around the iteration 200, HyDE still showed a performance of improvement after the 500 iterations, which could indicate that the capabilities for scaping of local optimums is better for this approach. 


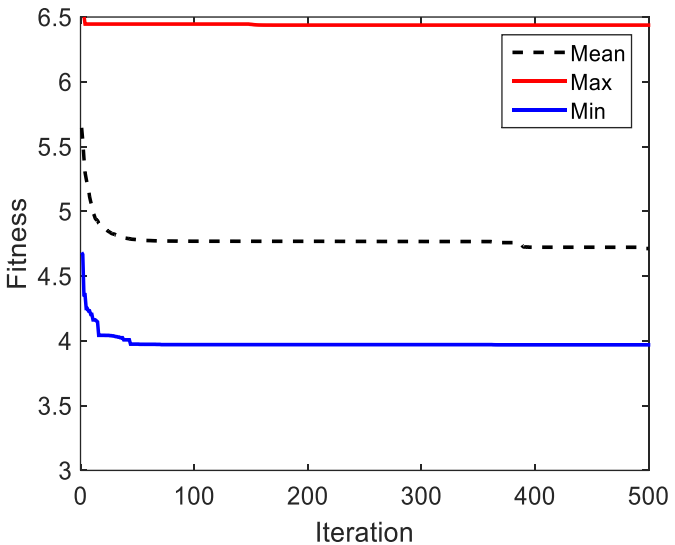

a)

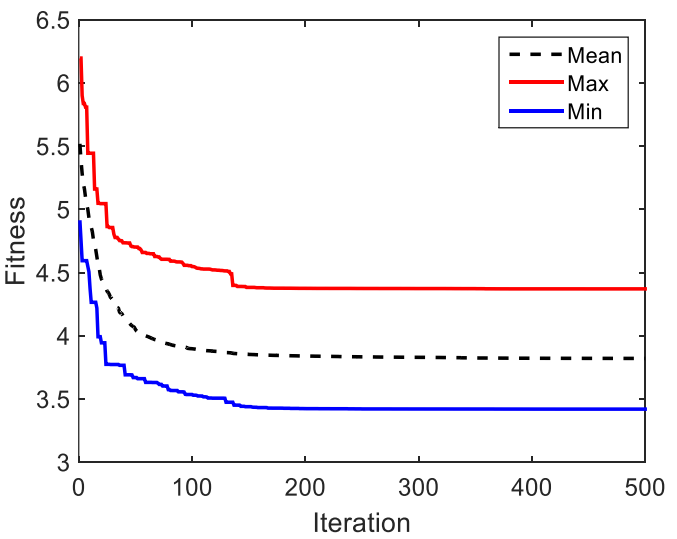

b)

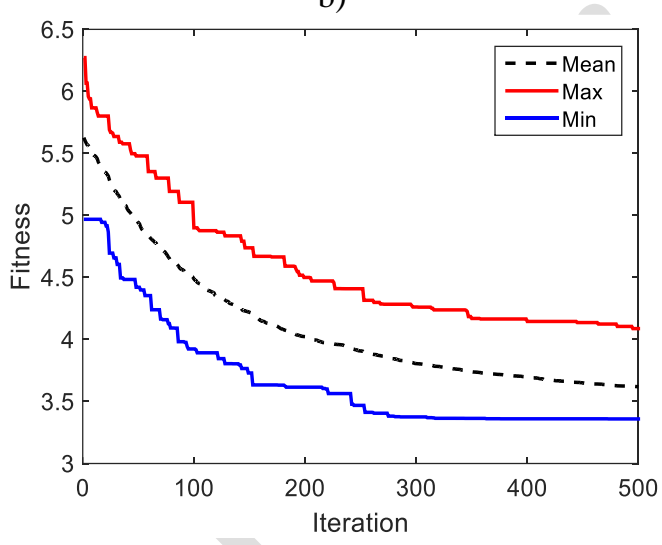

c)

Fig. 3 - Convergence behivor of the tested algorithms with a population of 10 individuals. a) PSO. b) DE. C) HyDE.

\section{CONCLUSIONS}

In this paper, an optimization problem to achieve DR in standalone mode is presented and solved using evolutionary computation. In particular, DE and one adaptive variant called HyDE are compared with the well-known PSO over a case study including batteries and flexible loads. The results showed that DE and its variant HyDE outperformed PSO for this application, showing better convergence capabilities and improving the fitness of the objective function. In fact, the evolutionary algorithms achieved a performance close to the value obtained by solving the problem with a deterministic approach using CPLEX. However, CPLEX is a commercial solver (not open source) that has a cost from practitioners to be used. In contrast, evolutionary algorithms are easy to implement in open source software (as python or java) highlighting their importance. Future work might explore realworld implementation or emulation of the approaches for effective DR applications.

\section{REFERENCES}

[1] B. Kirby, E. Ela, and M. Milligan, "Analyzing the Impact of Variable Energy Resources on Power System Reserves," in Renewable Energy Integration, vol. 13, no. 6, Elsevier, 2017, pp. 85-101.

[2] L. Park, Y. Jang, S. Cho, and J. Kim, "Residential Demand Response for Renewable Energy Resources in Smart Grid Systems," IEEE Trans. Ind. Informatics, vol. 13, no. 6, pp. 3165-3173, 2017.

[3] X. Zhou, J. Shi, Y. Tang, Y. Li, S. Li, and K. Gong, "Aggregate Control Strategy for Thermostatically Controlled Loads with Demand Response," Energies, vol. 12, no. 4, p. 683, Feb. 2019.

[4] J. Spínola, P. Faria, and Z. Vale, "Photovoltaic inverter scheduler with the support of storage unit to minimize electricity bill," Adv. Intell. Syst. Comput., vol. 619, pp. 63-71, 2017.

[5] B. Hussain et al., "A Weighted-Sum PSO Algorithm for HEMS: A New Approach for the Design and Diversified Performance Analysis," Electronics, vol. 8, no. 2, p. 180, Feb. 2019.

[6] R. Faia, P. Faria, Z. Vale, and J. Spinola, "Demand Response Optimization Using Particle Swarm Algorithm Considering Optimum Battery Energy Storage Schedule in a Residential House," Energies , vol. 12, no. 9. 2019.

[7] S. Das and P. N. Suganthan, "Differential Evolution: A Survey of the State-of-the-Art," IEEE Trans. Evol. Comput., vol. 15, no. 1, pp. 4-31, Feb. 2011

[8] S. Das, S. S. Mullick, and P. N. Suganthan, "Recent advances in differential evolution-An updated survey," Swarm Evol. Comput., 2016.

[9] "Differential Evolution," in Differential Evolution, Berlin/Heidelberg: Springer-Verlag, 2005.

[10] O. Kilkki et al., "Local market reference architecture and business requirements, D1.1 of DOMINOES Project," http://dominoesproject.eu, 2018. [Online]. Available: http://dominoesproject.eu/wpcontent/uploads/2018/06/D1.1_DOMINOES_LocalMarketReferenceA rchitecture_v1.2 final.pdf.

[11] R. Faia, T. Pinto, Z. Vale, and J. M. Corchado, "Strategic Particle Swarm Inertia Selection for Electricity Markets Participation Portfolio Optimization," Appl. Artif. Intell., vol. 32, no. 7-8, pp. 745-767, Sep. 2018.

[12] R. Faia, T. Pinto, Z. Vale, and J. M. Corchado, "Hybrid particle swarm optimization of electricity market participation portfolio," in 2017 IEEE Symposium Series on Computational Intelligence, SSCI 2017 Proceedings, 2018, vol. 2018-Janua.

[13] F. Lezama, J. Soares, R. Faia, T. Pinto, and Z. Vale, "A New HybridAdaptive Differential Evolution for a Smart Grid Application Under Uncertainty," in 2018 IEEE Congress on Evolutionary Computation (CEC), 2018, pp. 1-8.

[14] V. Miranda, H. Keko, and A. Jaramillo, "EPSO: Evolutionary particle swarms," Stud. Comput. Intell., vol. 66, pp. 139-167, 2007.

[15] J. Brest, S. Greiner, B. Boskovic, M. Mernik, and V. Zumer, "SelfAdapting Control Parameters in Differential Evolution: A Comparative Study on Numerical Benchmark Problems," IEEE Trans. Evol. Comput., vol. 10, no. 6, pp. 646-657, Dec. 2006.

[16] ERSE, "Tarifas e Precos para a Energia Eletrica e outros servicos em 2019," 2019. [Online]. Available: http://www.erse.pt/pt/electricidade/tarifaseprecos/2019/Documents/Di retiva ERSE 13-2018 (Tarifas e Preços EE 2019).pdf. [Accessed: 06Feb-2019]. 